9 - ORIGINAL ARTICLE

EXPERIMENTAL SURGICAL INFECTIONS

\title{
Genotyping of multidrug-resistant strains of Pseudomonas aeruginosa isolated from burn and wound infections by ERIC-PCR ${ }^{1}$
}

\author{
Azar Dokht Khosravi', Hajar Hoveizavi ${ }^{\mathrm{II}}$, Ali Mohammadian ${ }^{\mathrm{III}}$, Abbas Farahani ${ }^{\mathrm{IV}}$, Atefeh Jenabi ${ }^{\mathrm{III}}$ \\ DOI: http://dx.doi.org/10.1590/S0102-865020160030000009 \\ ${ }^{I} \mathrm{MD}$, Health Research Institute, Infectious and Tropical Diseases Research Center, Ahvaz Jundishapur University of Medical Sciences, Ahvaz, Iran. \\ Conception and design of the study, critical revision, final approval of the version to be published. \\ IIMD, Health Research Institute, Infectious and Tropical Diseases Research Center, Ahvaz Jundishapur University of Medical Sciences, Ahvaz, Iran. \\ Conception and design of the study, manuscript writing. \\ IIIMD, Department of Microbiology, School of Medicine, Ahvaz Jundishapur University of Medical Sciences, Ahvaz, Iran. Acquisition of data, technical \\ procedures. \\ ${ }^{\mathrm{IV}} \mathrm{MD}$, Department of Microbiology, School of Medicine, Ahvaz Jundishapur University of Medical Sciences, Ahvaz, Iran. Analysis and interpretation \\ of data.
}

\begin{abstract}
PURPOSE: To determine the genetic diversity of MDR P. aeruginosa strains isolated from burn and wound infections in Ahvaz, Iran, by ERIC-PCR.

METHODS: From total 99 strains of $P$. aeruginosa defined as MDR by using drug susceptibility testing, 66 were subjected to ERICPCR analysis, comprises 53 strains isolated from burn infection, and 13 randomly selected strains from wound infection with higher resistance to combinations of more numbers of drugs.

RESULTS: Eight clusters (I to VIII), and 50 single clones were generated for tested MDR isolates analyzed by ERIC-PCR. The high heterogeneity was observed among the isolates from burn infections including 16 isolates which were categorized in eight clusters and 37 single clones. The isolates in clusters II, III, VI, VIII showed $100 \%$ similarity.

CONCLUSIONS: The high level of genotypic heterogeneity in $P$. aeruginosa strains demonstrated no genetic correlation between them. Extremely high drug resistance in isolates from burn, suggests that efficient control measures and proper antibiotic policy should be observed.
\end{abstract}

Key words: Drug Resistance, Microbial. Polymerase Chain Reaction. Genotyping Techniques. Pseudomonas aeruginosa. 


\section{Introduction}

Pseudomonas aeruginosa is one of the most common causes of nosocomial infections which affects mainly immunocompromised hospitalized patients, and in particular, is the leading cause of life threatening infections in patients in burn units ${ }^{1-2}$. The clinical importance of $P$. aeruginosa is mostly related to its high resistance against antimicrobial drugs, and in addition to its intrinsic resistance to antibiotics, becomes quickly resistant against the drugs during the treatment ${ }^{3}$. The prevalence of multidrug-resistant (MDR) isolates has been increasing worldwide and poses a serious problem in hospital settings, with significant rise in patients' morbidity and mortality ${ }^{4}$. Previous studies show that the prevalence of MDR is dramatically increasing among $P$. aeruginosa isolates from burn and other hospitalized patients in $\operatorname{Iran}^{5-7}$.

Nowadays, different typing methods such as biotyping, serotyping, pyocin typing have been proposed to determine the common clones of $P$. aeruginos $a^{8}$. These clones and correlation between them are of importance in epidemiologic studies of $P$. aeruginosa nosocomial infections. However the discrimination power of these methods is lower compared to molecular typing system $^{4}$. So, in order to differentiate between the isolates and clonal groups of $P$. aeruginosa, several molecular typing schemes have been described ${ }^{9}$. These methods include pulsed field gel electrophoresis (PFGE), ribotyping, enterobacterial repetitive intergenic consensus polymerase chain reaction (ERIC-PCR) and random amplification of polymorphic DNA (RAPD). Among them, the main typing method with wider application is PFGE known as gold standard genotypic technique, though being high specific and sensitive, is limited by technical complexity, expense and prolonged turnaround times for results ${ }^{10-11}$.

One of the widely used repetitive DNA elements in PCRbased genotyping methods, are the ERIC sequences common to Gram-negative enteric bacteria ${ }^{10}$. ERIC sequences may offer greater potential as an example for the study of the evolution of bacterial interspersed repetitive sequences because they are longer and thus more informative in comparative analyses and are found in a wider range of species ${ }^{12}$. The ERIC -based PCR genotyping (ERIC-PCR) method has already been used to study the epidemiology of $P$. aeruginosa in Australia and Brazil ${ }^{8,13}$.

Since the rapid and discriminative subtyping methods are useful for determining the clonality of the strains in nosocomial outbreaks, the present study was designed to investigate the genetic diversity in a collection of the MDR strains of $P$. aeruginosa isolated from burn and wound infections by ERIC-PCR. The determination of genetic relationships among the MDR strains, allows mapping the dynamics of infection transmission in the region of the study and will also provides a better understanding the epidemiology of the resistant strains.

\section{Methods}

This study has been approved by the Infectious and Tropical Diseases Research Center.

\section{Bacterial strains}

From total 99 MDR strains, 66 were subjected to genotyping. These were came from 150 Pseudomonas aeruginosa isolates which were originated from wound and burn infections in Ahvaz, Iran, over a period of six months from January to November 2013, and the details are under publication ${ }^{14}$. The MDR determination was according to the criteria described by other investigators ${ }^{15}$.

\section{DNA extraction}

DNA extracted from colonies of all isolates by simple boiling method as described elsewhere ${ }^{16}$. In brief, a few colonies were dissolved in TE (Tris-EDTA) buffer and boiled at $100^{\circ} \mathrm{C}$ for 10 minutes with subsequent precipitation in a $14000 \mathrm{x} g$ refrigerated centrifuge at $4^{\circ} \mathrm{C}$ for $3 \mathrm{~min}$. The supernatant containing DNA was used as template for PCR amplification. The concentration of extracted DNA was measured by biophotometer (Eppendorf, Germany) at 260-280 nanometer and were kept at $-20^{\circ} \mathrm{C}$ until use.

\section{ERIC PCR fingerprinting}

PCR amplification was performed on extracted DNA by using previously reported primers of ERIC1 (5'-ATGTAAGCTCCTGGGGATTCAC-3') and ERIC2 (5'-AAGTAAGTACTGGGGTGAGCG-3' ${ }^{\prime}{ }^{17}$, in an Eppendorf thermocycler (Roche Co, Germany). PCR mixture was prepared in a final volume of $50 \mu \mathrm{l}$ containing $10 \mu \mathrm{l}$ of 10x PCR buffer, 50 $\mathrm{mM} \mathrm{MgCl}, 2.5 \mathrm{mM}$ dNTP mix, $10 \mu \mathrm{M}$ of each primer,1U of Taq DNA polymerase and $50 \mathrm{ng}$ of template DNA. The amplification parameters were initial denaturation at 94 for $3 \mathrm{~min}$, followed by 35 cycles of PCR consisting denaturation at 94 for $1 \mathrm{~min}$, annealing at 55 for $7 \mathrm{~min}$, and extension at 72 for $2 \mathrm{~min}$ and a final extension at $72^{\circ} \mathrm{C}$ for $5 \mathrm{~min}$. The amplified products were subjected to electrophoresis on $1.5 \%$ agarose gel, stained with 0.5 $\mu \mathrm{g} / \mu \mathrm{l}$ ethidium bromide (Qiagen, Germany) and analyzed under UV light in a gel documentation system (Proteinsimp, USA). Pseudomonas aeruginosa ATCC 27853 was used as positive 
control. Each ERIC-PCR test was performed in duplicate to ensure conformity of each fingerprint.

\section{ERIC Fingerprint analyses and dendrogram con- struction}

A TIFF image of gel was created by photographed with UV gel Doc (BIO-RAD, USA) and the DNA banding patterns entered into a database in BioNumerics 7.1 software (Applied Maths, Sint-Martens-Latem, Belgium). The ERIC-PCR patterns obtained were interpreted and compared as described by Ghazi et $a l .{ }^{18}$. Similarity analysis was calculated using the Dice coefficient and the unweighted pair group average (UPGMA) for cluster analyses. The criterion for related clones or classified as the same type when the pattern similarity was $80 \%$ or more similar bands.

\section{Results}

According to criteria determined for MDR $P$. aeruginosa ${ }^{15}$, 99 strains $(66 \%)$ were identified as MDR, of which $53(70.66 \%)$ were belonged to burn and $46(61.33 \%)$ were originated from wound infections. These isolates showed more than $90 \%$ resistance to the most of tested antibiotics (Table 1). From total 99 MDR isolates, 66 were subjected to ERIC-PCR analysis. These were included all 53 isolates from burn patients, which were showed resistance to at least nine of antibiotics, and 13 randomly selected from wound infections with the resistance patterns similar to those isolated from burn infections.

TABLE 1 - Disk diffusion antimicrobial resistance patterns of $150 P$. aeruginosa isolates. The isolates designated as MDR are among the most resistant isolates.

\begin{tabular}{ccc}
\hline Antibiotic & Disk content $(\boldsymbol{\mu g})$ & Resistant N. $(\%)$ \\
\hline ofloxacin & 5 & $110(73.33)$ \\
ciprofloxacin & 5 & $106(70.67)$ \\
gentamicin & 10 & $103(68.66)$ \\
imipenem & 10 & $93(62)$ \\
meropenem & 10 & $93(62)$ \\
ceftazidime & 30 & $102(68)$ \\
amikacin & 30 & $83(55.33)$ \\
aztreonam & 30 & $100(66.67)$ \\
ceftriaxone & 30 & $105(70)$ \\
cefotaxime & 30 & $109(72.67)$ \\
cefepime & 30 & $119(79.33)$ \\
ticarcillin & 75 & $106(70.67)$ \\
piperacillin & 100 & $68(45.33)$ \\
carbenicillin & 100 & $107(71.34)$ \\
\hline
\end{tabular}

Figure 1 represents the ERIC-PCR profiles of some $P$. aeruginosa strains isolated from burn infection by gel electrophoresis. Eight clusters were generated for analyzed MDR isolates, which were designated as: I, II, III, IV, V, VI, VII, VIII, and 50 single clones. In clusters II, III, VI and VIII; the isolates showed $100 \%$ similarity, and each of them has formed one real clone. All MDR isolates from wound infections showed heterogeneity as single clone.

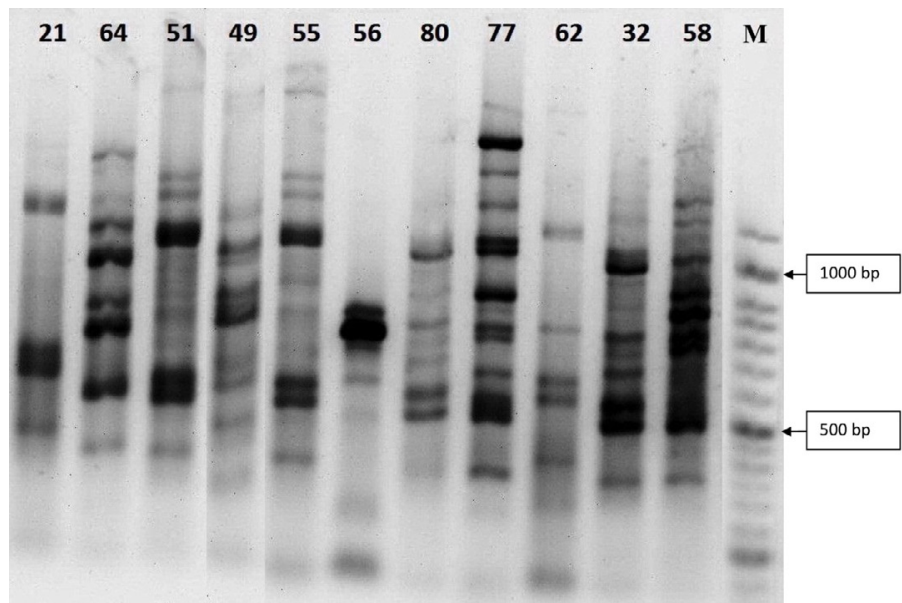

FIGURE 1 - DNA-banding profiles of some $P$. aeruginosa strains isolated from burn infection by gel electrophoresis following ERIC-PCR.

In Figure 2, the dendrogram generated on the basis of the isolates antimicrobial resistance against 14 main antibiotics are presented. The high unusual heterogeneity was observed among the isolates from burn infections, representing 16 isolates which were categorized in eight clusters (each cluster comprised 2 identical isolates), and 37 single clones. The members classified within the clusters, demonstrated highest resistance to more antibiotic categories in present study. 
ERIC-PCR

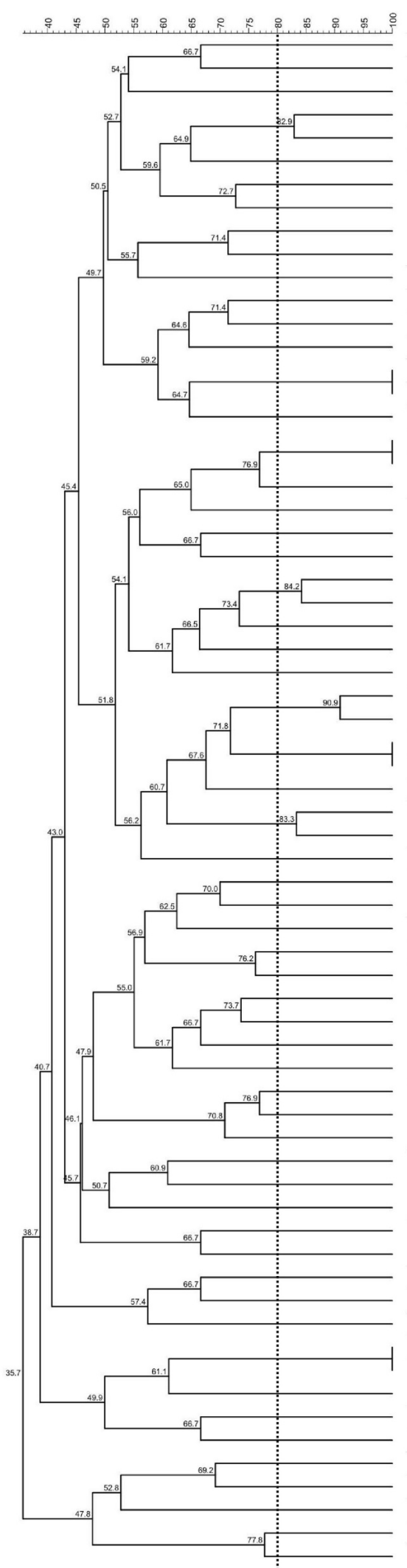

ERIC-PCR

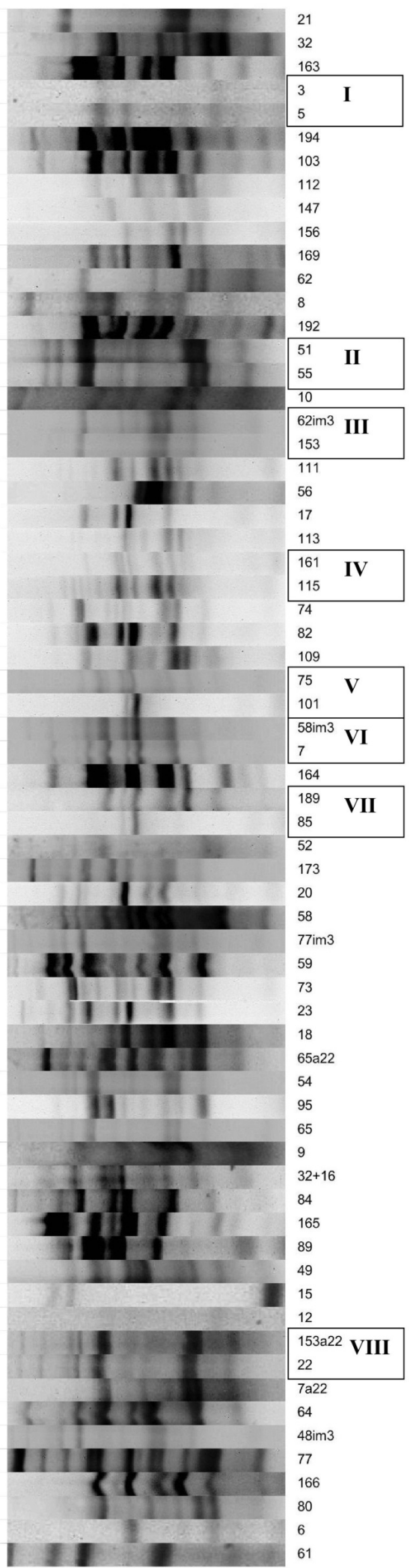

FIGURE 2 - Dendrogram of Pseudomonas aeruginosa based on ERIC-PCR. The isolates from both burn and wound infections. The scale at the top represents the genetic distance between the isolates. 


\section{Discussion}

Pseudomonas aeruginosa has a remarkable ability to acquire and harbor diverse resistance determinants ${ }^{19}$. Due to its intrinsic and acquired antimicrobial resistance, only limited classes of antibiotics are effective for the treatment of $P$. aeruginosa infection, so outbreaks due to MDR $P$. aeruginosa infection in hospitals despite efficient infection control policies, occurs worldwide ${ }^{20-21}$. The Taleghani burn hospital in Ahvaz, the center of Khuzestan province, is a referral center in Southwestern Iran, serves patients from neighboring provinces. Therefore genotyping of $P$. aeruginosa isolates seems to be essential to clarify the epidemiology of this organism in this hospital. In order to characterize the diversity of MDR strains of $P$. aeruginosa isolated from burn patients, and comparison with the heterogeneity of isolates from wound infections, $66 \mathrm{MDR}$ strains were subjected to ERIC-PCR analysis. These isolates which were comprises all MDR strains isolated from burn infection $(n=53)$ and 13 randomly selected strains from wound infections, were more resistant to combinations of very high numbers of drugs. With ERIC-PCR, the 66 isolates were sub-typed into eight different cluster profiles and 50 single clones. The clusters consisted only 16 isolates from burn infection and none of the wound isolates were included in the clusters. The majority of burn isolates and all those from wound infections, showed the diversity as single clones, which shows a high genotypic variation among the tested isolates. In a study conducted on 75 P. aeruginosa isolates from various sources, weak clonality was observed with $80 \%$ of the clinical strains belonging to only 3 clones $^{21}$, which was not in agreement with our study. Other investigators reported genetically diversity and heterogeneity among their 48 tested P. aeruginosa clinical isolates and concluded that both used analyses methods of ERICPCR and REP-PCR have been described as equally effective in characterizing clinical isolates of $P$. aeruginos $a^{10}$. Although the study of Lim et $a .^{22}$, was against the mentioned work, as they found that ERIC-PCR was more discriminatory than REP-PCR. In their report, ERIC-PCR, was able to differentiate isolates that were not differentiated by REP-PCR. Moreover the molecular typing of the MDR $P$. aeruginosa isolates revealed 21 genetic profiles with predominance of three clones in another study ${ }^{23}$. In our study, high level of genetic diversity was observed among the isolates, demonstrating no genetic correlation between them. We could not find any significant differences between genotypic diversity of isolates from burn and wound infections, due to high rate of heterogeneity in both isolates. In agreement to our study, ERICPCR produced 53 different ERIC fingerprints for $P$. aeruginosa isolates in the study of Goudarzi et al. ${ }^{24}$, from Iran, 49 of which contained only 1 strain. Eight of the isolates had 100\% similarity, forming four real clones. Besides, Inacio et al. ${ }^{9}$, demonstrated a high level of genetic heterogeneity among their tested isolates using ERIC-PCR analysis.

This $P$. aeruginosa heterogeneity differences in several studies from different parts of the world, demonstrate the impact of environmental factors and the level of hospital hygiene on the distribution and genetic clonal formation variation. As the results demonstrated, our studied MDR $P$. aeruginosa clinical isolates were genetically diverse and heterogeneous, suggesting that multiple subtypes of the species are involved in infection. Moreover the findings of present work suggest that genotyping by ERIC-PCR, may play an important role in routine epidemiological surveillance, and in the identification of the source of transmission of $P$. aeruginosa in the hospitals. As previously reported ${ }^{25}$, the ability of ERIC-PCR assay to discriminate types, proved to be excellent, and can be used as first screening genotyping methods for typing of $P$. aeruginosa.

\section{Conclusions}

Despite the strain diversity among clinical isolates both from burn and wound infections in present study, extremely high level of drug resistance especially in isolates from burn, suggests that efficient and sustained control measures and proper antibiotic policy should be observed. Moreover the high level of genotypic heterogeneity in $P$. aeruginosa strains demonstrated by ERICPCR, showed no genetic correlation between them.

\section{References}

1. Floret N, Bertrand X, Thouverez M, Talon D. Nosocomial infections caused by Pseudomonas aeruginosa: exogenous or endogenous origin of this bacterium? Pathol Biol. 2009;57(1):9-12. PMID: 18848405.

2. Leseva M, Arguirova M, Nashev D, Zamfirova E, Hadzhyiski O. Nosocomial infections in burn patients: etiology, antimicrobial resistance, means to control. Ann Burns Fire Disasters. 2013;26(1):511. PMID: 23966892.

3. Tsutsui A, Suzuki S, Yamane K, Matsui M, Konda T and Marui E. Genotypes and infection sites in an outbreak of multidrug resistant Pseudomonas aeruginosa. J Hosp Infect. 2011;78:317-22. PMID: 21689862.

4. Cholley P, Gbaguidi-Haore H, Bertrand X, Thouverez M, Plésiat P, Hocquet D, Talon D. Molecular epidemiology of multidrug-resistant Pseudomonas aeruginosa in a French university hospital. J Hosp Infect. 2010;76:316-9. PMID: 20692070.

5. Farshadzadeh Z, Khosravi AD, Alavi SM, Parhizgari N, Hoveizavi H. Spread of extended-spectrum $\beta$-lactamase genes of blaOXA-10, blaPER-1 and blaCTX-M in Pseudomonas aeruginosa strains isolated from burn patients. Burns. 2014;40:1575-80. PMID: 24767142 . 
6. Moazami Goudarzi S, Eftekhar F. Multidrug resistance and integron carriage in clinical isolates of Pseudomonas aeruginosa in Tehran, Iran. Turk J Med Sci. 2015;45(4):789-93. PMID: 26422847.

7. Saderi H, Owlia P. Detection of multidrug resistant (MDR) and extremely drug resistant (XDR) $P$. aeruginosa isolated from patients in Tehran, Iran. Iran J Pathol. 2015;10(4):265-71. PMID: 26351496.

8. Stehling EG, Leite DS, Silveira WD. Molecular typing and biological characteristics of Pseudomonas aeruginosa isolated from cystic fibrosis patients in Brazil. Braz J Infect Dis. 2010;14(5):4627. PMID: 21221474.

9. Inacio HS, Bomfim MR, França RO, Farias LM, Carvalho MA, Serufo JC, Santos SG. Phenotypic and genotypic diversity of multidrug-resistant Pseudomonas aeruginosa Isolates from bloodstream infections recovered in the Hospitals of Belo Horizonte, Brazil. Chemotherapy. 2014;60(1):54-62. PMID: 25402327.

10. Syrmis MW, O'Carroll MR, Sloots TP, Coulter C,Wainwright CE, Bell SC, Nissen MD. Rapid genotyping of Pseudomonas aeruginosa isolates harboured by adult and paediatric patients with cystic fibrosis using repetitive-element-based PCR assays. J Med Microbiol. 2004;53:1089-96. PMID: 15496385.

11. Wolska K, Kot B, Jakubczak A. Phenotypic and genotypic diversity of Pseudomonas aeruginosa strains isolated from hospitals in siedlce (Poland). Braz J Microbiol. 2012;43(1):274-82. PMID: 24031829.

12. Wilson LA, Sharp PM. Enterobacterial repetitive intergenic consensus (ERIC) sequences in Escherichia coli: Evolution and implications for ERIC-PCR. Mol Biol Evol. 2006;23(6):1156-68. PMID: 16533821

13. Kidd TJ, Gibson JS, Moss S., Greer RM, Cobbold RN, Wright JD, Grimwood K, Bell SC. Clonal complex Pseudomonas aeruginosa in horses. Vet Microbiol. 2011;149:508-12. PMID: 21183294.

14. Khosravi AD, Mohammadian A. Efflux MexAB-mediated resistance in multidrug and pan-drug resistant strains of Pseudomonas aeruginosa isolated from patients with burn and wound infections. Jundishapur J Nat Pharm Prod. 2016. [Epub ahead of print].

15. Thong KL, Lai KS, Ganeswrie R, Puthucheary SD. Pulsed-field gel electrophoresis of multidrug-resistant and -sensitive strains of Pseudomonas aeruginosa from a Malaysian hospital. Jpn J Infect Dis. 2004;57:206-9. PMID: 15507777.

16. Lee S, Park YJ, Kim M, Lee HK, Han K, Kang CS, Kang MW. Prevalence of Ambler class A and D b-lactamases among clinical isolates of Pseudomonas aeruginosa in Korea. J Antimicrob Chemother. 2005;56:122-7. PMID: 15890715.

17. Tosin I, Silbert S, Sader HS. The use of molecular typing to evaluate the dissemination of antimicrobial resistance among Gram-negative rods in Brazilian hospitals. Braz J Infect Dis. 2003;7:360-9. PMID: 14636474

18. Ghazi M, Khanbabaee G, Fallah F, Kazemi B, Mahmoudi S, Navidnia M, Pourakbari B, Bakhshi B, Goudarzi H. Emergence of Pseudomonas aeruginosa cross-infection in children with cystic fibrosis attending an Iranian referral pediatric center. Iran J Microbiol. 2012;4(3):124-9. PMID: 23066486.

19. Hirsch EB, Tam VH. Impact of multidrug-resistant Pseudomonas aeruginosa infection on patient outcomes. Expert Rev Pharmacoecon Outcomes Res. 2010;10(4):441-51. PMID: 20715920.

20. Hong DJ, Bae IK, Jang IH, Jeong SH, Kang HK, Lee K. Epidemiology and characteristics of metallo- $\beta$-lactamase-producing Pseudomonas aeruginosa. Infect Chemother. 2015;47(2):81-97. PMID: 26157586.

21. Janam R, Gulati AK, Nath G. Antibiogram and genotyping of Pseudomonas aeruginosa isolated from human, animal, plant, water and soil sources in north India. Southeast Asian J Trop Med Public Health. 2011;42(6):1477-88. PMID: 22299419.
22. Lim KT, Yasin RM, Yeo CC, Puthucheary SD, Balan G, Maning $\mathrm{N}$, Wahab ZA, Ismail N, Tan EA, Mustaffa A, Thong KL. Genetic fingerprinting and antimicrobial susceptibility profiles of Pseudomonas aeruginosa hospital isolates in Malaysia. J Microbiol Immunol Infect. 2009;42(3):197-209. PMID: 19812853.

23. Jácome PR, Alves LR, Cabral AB, Lopes AC, Maciel MA. Phenotypic and molecular characterization of antimicrobial resistance and virulence factors in Pseudomonas aeruginosa clinical isolates from Recife, State of Pernambuco, Brazil. Rev Soc Bras Med Trop. 2012;45(6):707-12. PMID: 23295873.

24. Goudarzi H, Karimi F, Asadi Amoli F, Abedinyfar Z, Doustdar F, Mehrnejad F. Genetic fingerprinting and antimicrobial susceptibility profiles of Pseudomonas aeruginosa isolates from eye infections. Iranian J Clin Infect Dis. 2011;6(1):41-6.

25. Dawson SL, Fry JC, Dancer BN. A comparative evaluation of five typing techniques for determining the diversity of fluorescent pseudomonads. J Microbiol Methods. 2002;50(1):9-22. PMID: 11943354.

\section{Correspondence:}

Hajar Hoveizavi

Health Research Institute, Infectious and Tropical Diseases Research Center

Ahvaz Jundishapur University of Medical Sciences

Ahvaz, Iran

Phone: +98 6133738392

hajarhoveizavi94@gmail.com

Received: Nov 21, 2015

Review: Jan 18, 2016

Accepted: Feb 16, 2016

Conflict of interest: none

Financial source: Ahvaz Jundishapur University of Medical Sciences (Grant No. 91132)

${ }^{1}$ Research performed at Health Research Institute, Infectious and Tropical Diseases Research Center, Ahvaz Jundishapur University of Medical Sciences, Ahvaz, Iran 